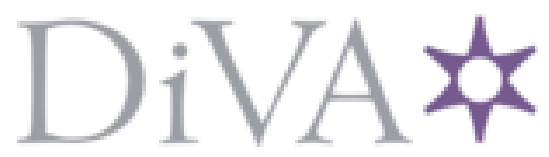

http://www.diva-portal.org

\title{
Preprint
}

This is the submitted version of a paper presented at IEEE PowerTech Conference Eindhoven.

Citation for the original published paper:

Singh, R S., Hooshyar, H., Vanfretti, L. (2015)

Laboratory Test Set-up for the Assessment of PMU Time Synchronization Requirements.

In: IEEE conference proceedings

http://dx.doi.org/10.1109/PTC.2015.7232731

N.B. When citing this work, cite the original published paper.

Permanent link to this version:

http://urn.kb.se/resolve?urn=urn:nbn:se:kth:diva-175858 


\section{Laboratory Test Set-up for the Assessment of PMU Time Synchronization Requirements}

\author{
Ravi Shankar Singh \\ Aalto University \\ Espoo, Finland \\ Email: ravi.singh@aalto.fi
}

\author{
Hossein Hooshyar \\ KTH Royal Institute of Technology \\ Stockholm, Sweden \\ Email: hossein.hooshyar@ee.kth.se
}

\author{
Luigi Vanfretti \\ KTH Royal Institute of Technology \\ Stockholm, Sweden \\ Statnett SF, Oslo \\ Oslo, Norway \\ Email: luigi.vanfretti@ee.kth.se
}

\begin{abstract}
This paper presents the construction of a Hardwarein-the-loop laboratory test set-up designed to assess the time synchronization requirements of Phasor Measurement Units (PMUs). The test set-up also allows to investigate the effects of signal phase shifts caused by current and voltage transformers, errors in timing source and the impact of these errors when determining time synchronization compliance of PMUs. The paper also describes the structure of an IRIG-B time-sync realtime implementation, which allowed real-time hardware-in-theloop simulation by providing a PMU with a high-accuracy timing source.
\end{abstract}

Index Terms-Phasor Measurement Unit (PMU), Real-Time Simulation, Hardware-in-the-Loop (HIL), IRIG-B.

\section{INTRODUCTION}

\section{A. Motivation}

Phasor Measurement Units (PMUs) are nowadays being used to generate time synchronized phasor measurements used in wide-area monitoring, control and protection systems for the modern power grid [1]. Logged synchrophasor records also help in event reconstruction after disturbances or failures take place in the grid. This helps in detailed examination of the events leading up to a disturbance [2]. To obtain reliable phasor estimates from a PMU, the instrument should be supplied with a high-accuracy timing source. The timing source should accurately synchronize the PMU's clock with the Coordinated Universal Time (UTC) which serves as a common reference for all PMUs in the grid [3].

As shown in Fig. 1, inaccuracies in PMU timing will degrade PMU phasor estimates. In the figure, a signal $\mathrm{X}$ is continuously sampled by two PMUs to obtain phasor estimates. If the timing of PMU2 is offset by ' $t$ ' seconds and the estimated phasors of both the PMUs are plotted in phasor domain, then both phasors will differ by a certain amount of phase error. This phase error will present itself in the phasor estimate of the PMU whose timing was incorrect. A timing error of $5 \mathrm{~ms}$ can cause an error of $\pm 90^{\circ}$ in the estimated phasor at a system frequency of $50 \mathrm{~Hz}$. The error in estimated phasor by PMU can be quantified in terms of the Total Vector

This work is supported by the FP7 IDE4L project funded by the European Commission, the STandUp for Energy Collaboration Initiative and by Statnett $\mathrm{SF}$, the Norwegian TSO).
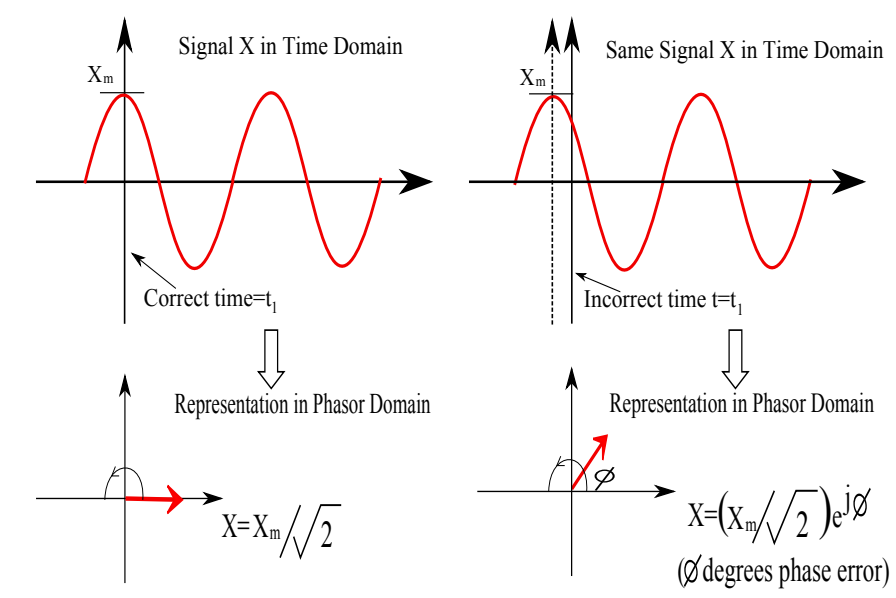

Fig. 1: Importance of time in correct phasor estimation

Error (TVE). The TVE is an expression of the difference between the theoretical value and the PMU's estimate of the signal at the same time [4]. So PMU timing errors will affect the TVE directly. As stated in the IEEE Standard C37.118.12011 for Synchrophasors Measurements for Power Systems, the timing source should be accurate enough to keep the TVE within $1 \%$ [4].

\section{B. Previous Work}

Previous works have focused on assessing the TVE compliance criteria of PMUs under steady-state conditions [5]. However, these tests were performed using stand-alone relay test kits. These test kits supply current and voltage signals directly to the PMUs. In practical applications of commercial PMUs, these signals are fed to the PMUs via instrument channel consisting of current and voltage transformers (CTs and VTs). This instrument channel introduces ratio and phase errors in the voltage and current signals before feeding them to the PMUs [6]. The errors will affect the final TVE of the PMU. As shown before, PMU timing accuracy will directly affect the phase errors of the PMU. As the total TVE should be less than $1 \%$, the share of TVE due to timing source inaccuracy need to decrease in the presence of errors caused by the instrumentation channel. This requires the reassessment 
of timing requirements for PMUs. Note that the test set-up mentioned above did not include CTs and VTs and hence will only be suitable for the ideal case where it is assumed that the PMU is getting error free input signals.

\section{Contributions}

This paper proposes a method and presents a real-time, Hardware-in-the-Loop (HIL) test set-up to assess PMU timing requirements. It also shows that the timing requirement in terms of accuracy for commercial PMUs should be stricter than the $31.8 \mu \mathrm{s}$ limit set in the IEEE Standard C37.118.12011.

The paper is organized as follows. Section II presents the tests carried out in previous works using stand-alone relay test kits. Section III discusses the concept of the proposed test and then discusses the set-up for real-time HIL test to access the PMU timing requirements in a laboratory. Some results in brief are presented in section IV and conclusions are drawn in section $\mathrm{V}$.

\section{STAND-ALONE PMU TEST}

This section briefly outlines the stand-alone test set-up to test the steady-state compliance of PMUs. To estimate phasors reliably, a PMU requires two inputs, power system signals (V \& I) and a high accuracy timing source. To test the performance of a PMU in the laboratory, sources to generate both inputs are required. In this stand-alone test, standard voltage and current signals are injected into the PMUs using stand-alone relay test kits such as Freja 300 [7]. A highaccuracy time signal is supplied using a Global Positioning System (GPS)-assisted substation clock (Arbiter 1094b [8]). Phasor estimates generated by the PMUs are sent to Phasor Data Concentrator (PDC). The set-up is shown in Fig. 2.

The measured values of the signals are compared to the original signal and the TVE and phase error is calculated from the logged PMU data. This phase error in the PMU phasor estimate is caused by the PMU's signal processing unit (which includes Analog to Digital converters and step down transformers) [2]. This test set-up does not include the instrumentation channel, and therefore, the PMU estimate is not affected by any kind of external phase errors caused by CTs and VTs. It is known that phase angle error for VTs is in the range of $\pm 4^{\circ}$ and for CTs in the range of $\pm 2^{\circ}$. In high accuracy instrument transformers, the phase angle varies between $\pm 0.1^{\circ}$ [9]. The output voltage from the VTs lags the original voltage signal and the current output form the CTs leads the original current signal as shown in Fig. 3. These phase shifts will affect the accuracy of phasor estimates generated by a PMU, thus directly affecting the TVE also.

A $1 \%$ TVE corresponds to a phase angle error of $0.573^{\circ}$, provided no other errors are present. This translates to $31.8 \mu \mathrm{s}$ in time at a power system frequency of $50 \mathrm{~Hz}$ (or $26 \mu$ s at $60 \mathrm{~Hz}$ ). The presence of phase errors in input signals reduces the margin for time source inaccuracies to be less than the 31.8 $\mu \mathrm{s}$ limit specified in IEEE C37.118.1-2011 [4]. The next section presents a method and HIL test set-up to study and

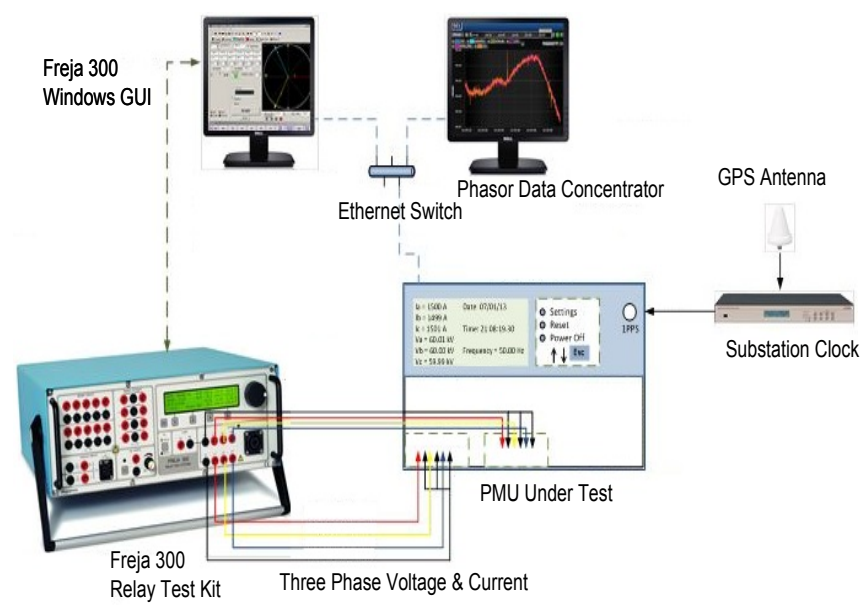

Fig. 2: PMU test using stand-alone relay test kit
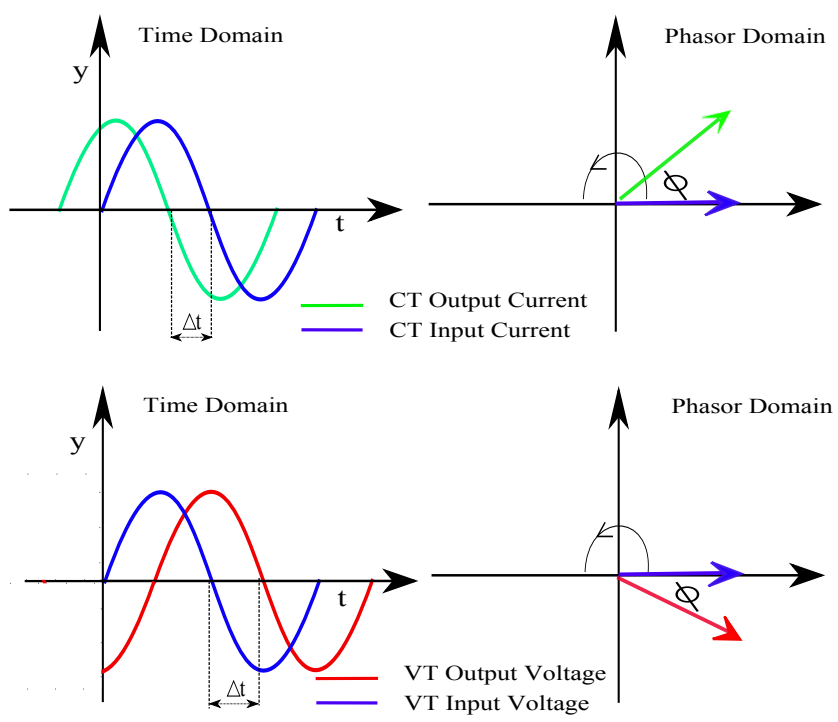

Fig. 3: Phase shift in current and voltage signals due to CTs and VTs

assess the time synchronization requirements of PMUs in a controlled laboratory environment.

\section{Test Set-Up for Assessment of PMU Time SYCNHRONIZATION REQUIREMENTS}

This method assesses the timing requirements of PMUs when the required accuracy of the timing source is of concern. For a fair assessment, it is necessary to test the PMU in an environment emulating the on-field implementation of a PMU. On field, the PMU is supplied with signals via an instrumentation channel. Also, to see the effect of varying timing errors on the TVE and hence estimate the acceptable accuracy limits of the timing source, a controllable PMU timing solution is required. This timing solution must be able to supply time with varying accuracy as required. In the case of above mentioned stand-alone test, there is no instrumentation channel and also the time clock supplying the time to PMU is assisted by GPS and hence is uncontrollable. The test set- 
up discussed in the paper tests the PMUs in presence of an emulated instrumentation channel and using a user controllable time source.

To emulate the instrumentation channel, Megger amplifiers SMRT1 were placed in between the current and voltage signals generation source and the PMU under test. The phase angle accuracy of the amplifier's output current and voltage signals could be up to $\pm 0.25^{\circ}$ at $50 \mathrm{~Hz}$ [10]. This accuracy range was found suitable as a substitute to emulate high accuracy instrument transformers whose phase angle accuracy varies between $\pm 0.1^{\circ}$.

To be able to control the PMU timing signal, it was decided to simulate the time signal in real-time and then feed it to the PMU. Basic control blocks from SIMULINK's library can be used to vary the accuracy of the time signal supplied. An InterRange Instrumentation Group Code B (IRIG-B) time signal was chosen as the high accuracy PMU timing source [11]. It distributes time to PMUs with an accuracy of $\pm 500 \mathrm{~ns}$ [12] and can be simulated and controlled using SimULINK. The PMU under test also requires balanced three-phase voltage and current signals as inputs. Both signals required for the test, i.e. IRIG-B timing signal and the three-phase voltage and current (V and I) signals, can be simulated in SIMULINK. These signals can be sent in real-time as inputs to the PMU. The structure of IRIG-B time code which was simulated in real-time for PMU time synchronization is briefly discussed below.

\section{A. IRIG Overview}

IRIG-B is a serial time code developed by the Telecommunication Group of IRIG. IRIG has six different encoding formats labelled A, B, D, E, G and $H$, out of which IRIG-B is most commonly used to distribute time to Intelligent Electronic Devices (IEDs) [11]. IRIG-B can be distributed in two ways, one as a DC Level Shifted (DCLS) pulse width coded signal (unmodulated) or as an amplitude modulated signal based on a sine wave carrier with a frequency of $1 \mathrm{kHz}$ [13]. GPS based clocks can generate IRIG-B coded timing signals which can be distributed to many IEDs in single substation. Unmodulated DCLS IRIG-B can achieve accuracies in the range of $\pm 500 \mathrm{~ns}$., which is better than the accuracy of $\pm 10 \mu s$ achievable by its amplitude modulated counterpart [12]. This makes the DC shifted, unmodulated form of IRIG$\mathrm{B}$, the most suitable format for time distribution in PMU applications.

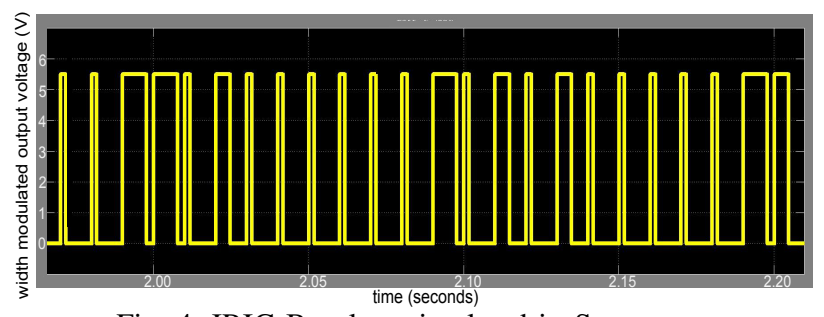

Fig. 4: IRIG-B pulses simulated in SIMULINK

\section{B. Unmodulated DCLS IRIG-B}

Unmodulated DCLS IRIG-B is distributed in frames. Each frame is one second long and has a pulse rate (or a bit rate) of 100 pulses per second (pps). The 'on-time' for each bit refers to the leading edge of the pulse. Each bit has an index count identification number. The Index count interval is the time interval between the leading edges of two consecutive pulses. Each bit has an index interval of 10ms. [13]. The index count ranges from 0 to 99 and then rolls over to 0 for the next second. Each frame uses five types of pulses: logic zero, logic one, position markers $\mathrm{P}_{0}-\mathrm{P}_{9}$, a reference bit $\mathrm{P}_{\mathrm{r}}$ and index markers. Reference bit $\mathrm{P}_{\mathrm{r}}$ indicates start of a new frame. Position identifiers from $\mathrm{P}_{0}$ through $\mathrm{P}_{9}$ are placed every ten bits. Index markers occur between decimal digits in each sub-word to provide visual separation. The width of these pulses are pulse width coded where the width of logic zero is set to be $20 \%$ of the index interval $(2 \mathrm{~ms})$, the width of logic one is $50 \%$ of the index interval $(5 \mathrm{~ms})$. Position markers and the reference bit are $80 \%$ of the index interval $(8 \mathrm{~ms})$ and index marker bits are $20 \%$ of the index interval $(2 \mathrm{~ms})$. Every new one-second time frame is identified by two consecutive $8 m s$ pulses, $\mathrm{P}_{0}$ and $\mathrm{P}_{\mathrm{r}}$. The structure of an IRIG-B frame is presented below:

$<$ synch $>$ SS:MM:HH:DDD $<$ Control $><$ Binary Seconds $>$ where:
$S S$

$M M$

$H H$

$D D D$

Control

Binary Seconds
The second of the minute [00 to 59 (60 during leap seconds)] in Binary Coded Decimal (BCD)

The minute of the hour (00 to 59) in BCD

The hour of day (00 to 23) in BCD The day of year (001 to 366) in BCD Informations like leap second, daylight saving, quality information and a parity bit included in a block of 27 bits Second of the day in 17 bits
To be able to use IRIG-B as a controllable timing source for PMUs under test, MATLAB code was written to generate pulse width modulated IRIG-B signals in the DCLS format specified above. The code was embedded in a SIMULINK model as a MATLAB function. The code includes an option to set the initial time from which time starts rolling. Other control parameters like daylight saving, leap seconds and time quality can also be set and changed. The parameters giving initial time of the IRIG-B time block can be set as shown in Fig. 5.

\section{Real-Time Hardware-in-the-loop set-up}

For this test set-up, real-time voltage and current signals and real-time and controllable IRIG-B timing signals were required as inputs to the PMUs under test. All these signals use the same time reference. These signals were obtained by executing the IRIG-B and balanced three phase current and voltage signals SimULINK blocks on the Opal-RT's eMEGASIM real- 


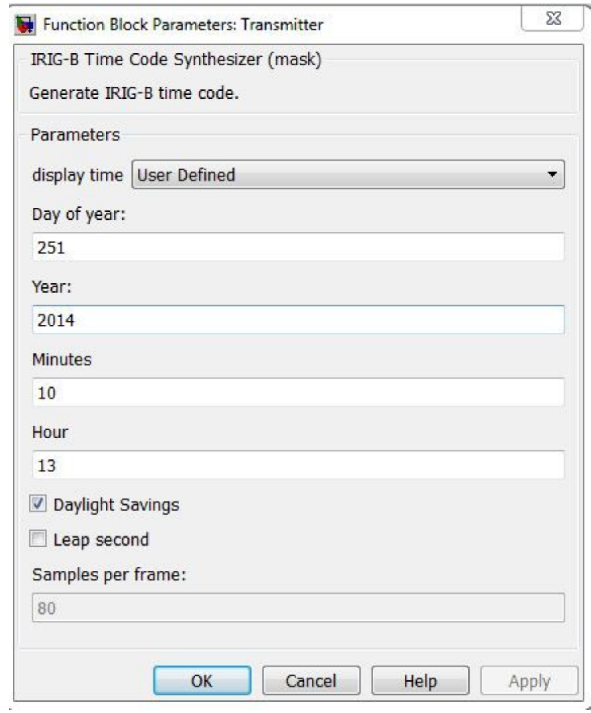

Fig. 5: Initial parameters for IRIG-B generator block in SIMULINK

time simulator platform [14]. Analog pulses of IRIG-B and analog voltage and current signals were obtained from the simulators analog output terminals. The simulator can produce analog outputs up to $\pm 16 \mathrm{~V}$. As the commercial PMUs required IRIG-B time code pulse of $5.5 \mathrm{~V}$ amplitude, the analog IRIG-B pulses were generated in real-time with amplitude of $5.5 \mathrm{~V}$ and were directly sent to the PMU IRIG-B input. This synchronized the PMUs to the time specified by the user as the initial parameters. The rated voltage and current inputs of the commercial PMUs were higher than the signals generated by the real-time simulator, i.e. $\pm 16 \mathrm{~V}$. Thus, the three phase voltage and current signals generated by the simulator were amplified using the Megger SMRT1 amplifiers and then were fed to the voltage and current inputs of the PMU. In this set-up, the Megger amplifiers emulated on-field CTs and VTs as they similarly introduce phase angle shifts in the voltage and current signals. The whole channel from the real-time simulator's output to the PMU's current and voltage inputs including the amplifiers and the connection cables represents the instrumentation channel. The complete model-to-data analysis work-flow for this test is shown in Fig. 6 The green lines in the figure indicate transfer of data over Ethernet. The instrument channel is shown using the red arrows.

The PMU makes phasor estimates of the signals received. The timing signals can be manipulated by the user during the test. Basic blocks like 'Transmission Delay' from SimulinK's library can be used to delay the time signals for a few microseconds to a few milliseconds. In the experiment, the time errors were varied in the steps of $10 \mu \mathrm{s}$. Continuously generated synchrophasor estimates by the PMU for different timing errors were sent to the Phasor Data Concentrator (PDC) and stored. This logged data was analysed using MATLAB. The effect of timing errors on the phase errors of the measured signals and consequently on the overall TVE of the estimated phasor was studied and plotted on the graphs.

\section{RESULTS}

After analysing the recorded phasor data, it was realized that the phase error due to the instrumentation channel can cause a major rise in the final TVE. Fig. 7 shows the phase angle errors for voltage signals fed to a commercial PMU. It is observed that in presence of instrumentation channel errors the time error of $10 \mu \mathrm{s}$ caused the voltage phase angle errors cross the $0.573^{\circ}$ mark which breaches the $1 \%$ TVE limit. Therefore, it can be asserted that the errors due to the instrumentation channel and errors in the timing source should be considered while examining the timing requirements for commercial PMUs.

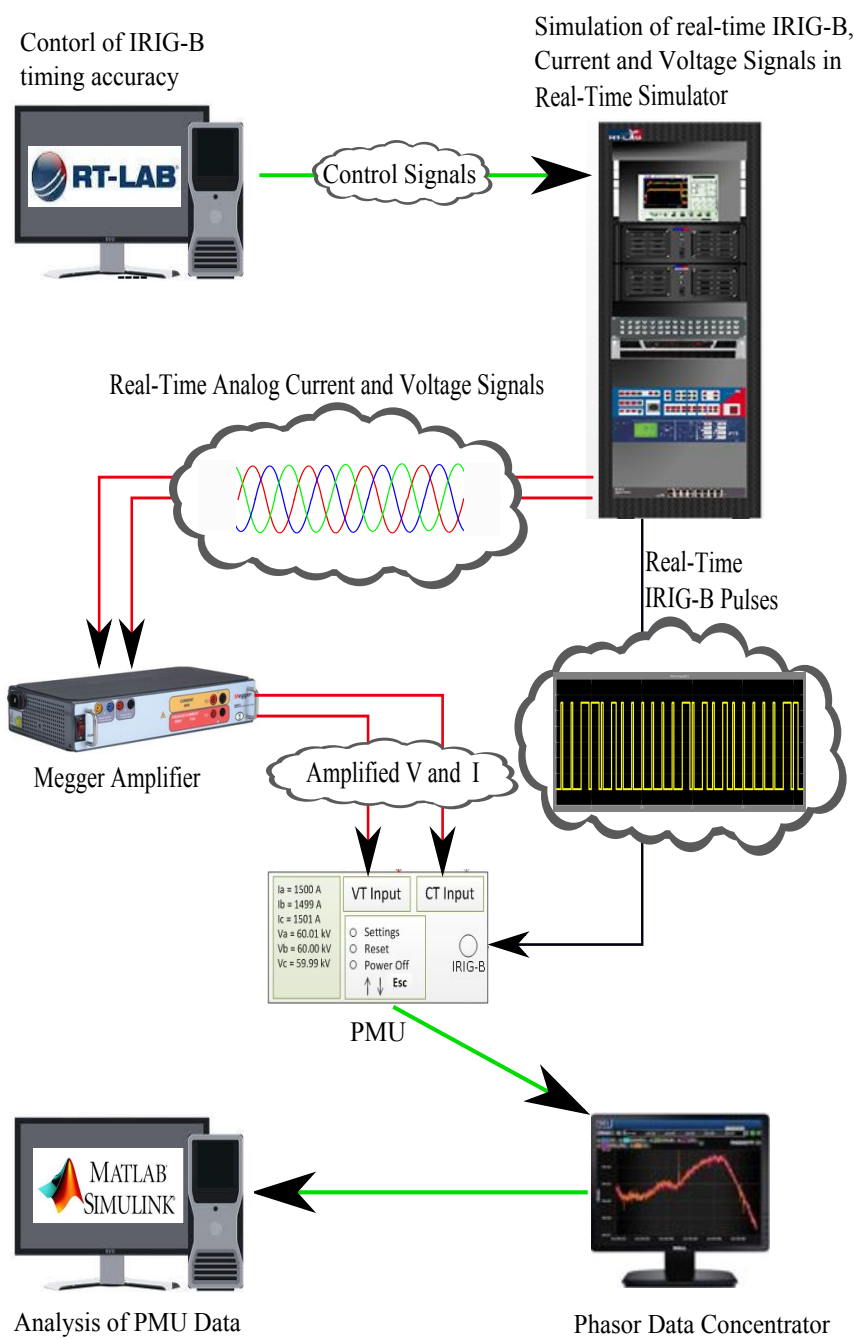

Fig. 6: Real-Time, Hardware-in-the-loop set-up to determine timing accuracy requirements 


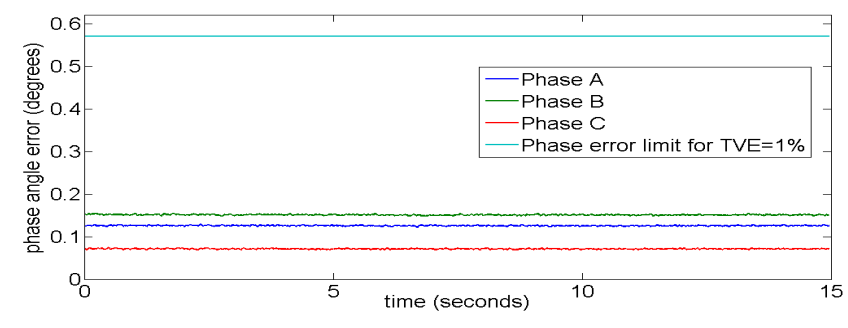

(a) Phase error in measured voltage signals in stand-alone tests

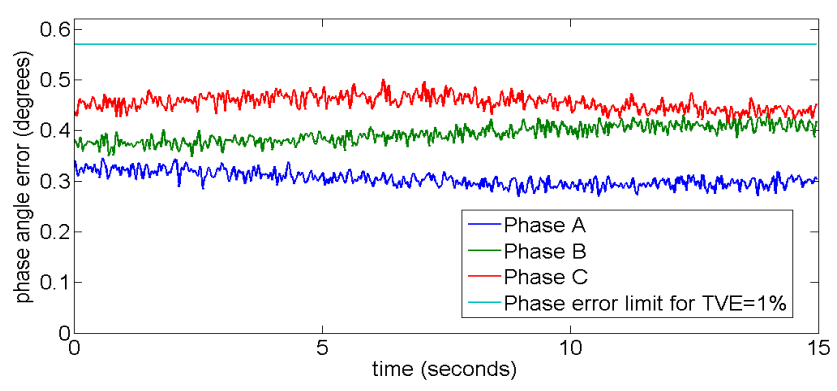

(b) Phase error in measured voltage signals in presence of Instrumentation Channel

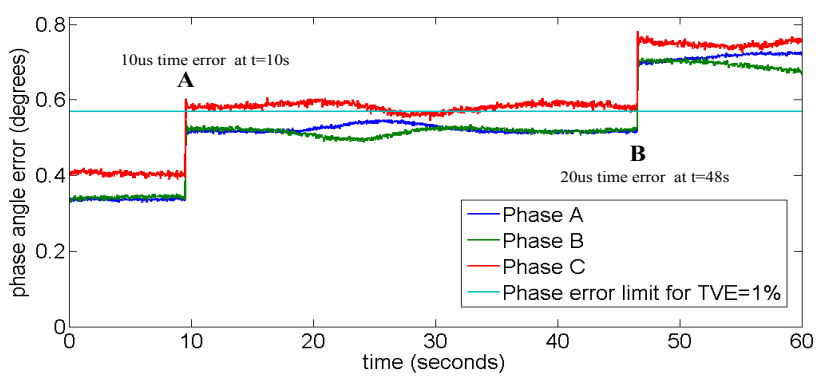

(c) Phase error in measured voltage signals in presence of instrumentation channel and varying time error (leading time) in steps of $10 \mu \mathrm{s}$ at point $\mathrm{A}$ and $\mathrm{B}$

Fig. 7: Phase angle variation in measured voltage for a commercial PMU for different test cases

\section{CONCLUSION}

This paper has detailed the development and implementation of a real-time test set-up to determine the timing accuracy requirements for PMUs. A generic hardware-in-the-loop setup was constructed using OPAL-RT's real-time simulation platform and commercial PMU units. A brief overview of IRIG-B as PMU's timing signals was also discussed.

Tests were performed using a controllable simulation of a timing source to explore the effects of timing errors on the Total Vector Error. Test results performed on a commercial PMU were discussed and it was observed that phase angle errors due to the instrumentation channel and the timing source can significantly affect the final TVE of the PMU measurement system. Hence the errors caused by the instrumentation channels should be taken into account while standardizing the PMU timing requirements. A detailed test results on two different commercial PMUs are discussed in a companion publication [15].

\section{REFERENCES}

[1] B. Vandiver, A. Apostolov, F. Stein Hauser, "Testing of Phasor Measurement Units," in 2010 63rd Annual Conference for Protective Relay Engineers, pp. 1-5, Apr. 2010.

[2] B. Dickerson, "Time in the Power Industry:How and Why We Use It" available online at:http://www.arbiter.com/files/product-attachments/ TimeInThePowerIndustry.pdf

[3] IEEE Guide for Synchronization, Calibration, Testing, and Installation of Phasor Measurement Units (PMUs) for Power System Protection and Control, C37.242-2013, March 2013.

[4] IEEE Standard for Synchrophasor Measurements for Power Systems, C37.118.1-2011, Dec 2011.

[5] M. S. Almas, J. Kilter and L. Vanfretti, "Experiences with steady-state PMU compliance testing using standard relay testing equipment", in Proc. Electric Power Quality and Supply Reliability Conference (PQ), 2014, pp. 103-110.

[6] Z. Wu, K. Thomas, R. Sun, V. A. Centeno and A. G. Phadke, "Threephase instrument transformer calibration with synchronized phasor measurements", in Proc. 2012 IEEE PES Innovative Smart Grid Technologies (ISGT), pp. 1-6.

[7] Megger, Freja 300 - Relay Test System, [Online]. Available: http://www. megger.com/eu/products/ProductDetails.php?ID=861\&Description=.

[8] Arbiter, GPS Substation Clock Model 1094B, [Online]. Available: http://www.arbiter.com/catalog/product/ model-1094b-gps-satellite-precision-time-clock.php

[9] Z.Huang, et al, "Performance evaluation of phasor measurement systems", in Proc. Power and Energy Society General Meeting - Conversion and Delivery of Electrical Energy in the 21st Century,2008, pp. 1-7.

[10] Megger, SMRT1, Single Phase Relay Tester, [Online]. Available: http: //www.megger.com/us/products/ProductDetails.php?ID=1529

[11] Jin-kai Huang, Liang Du and Qun-ying Liu, "Application of IRIG-B Code in Phase Measurement Unit", in 2012 Asia-Pacific Power and Energy Engineering Conference (APPEEC), pp. 1-3, Mar 2012.

[12] Jackie Peer, Eric Sagen, Shankar Achanta and Veselin Skendzic, "The Future of Time: Evolving requirements for Precize Time Synchronization in the Electric Power Industry," Schweitzer Engineering Laboratories, Inc., 2011, [Online]. available: https://www.selinc.com/WorkArea/ DownloadAsset. aspx ?id=9995

[13] IRIG Standard 200-98,IRIG Serial Time Code Formats, May 1998. [Online]. Available: http://www.irigb.com/pdf/wp-irig-200-98.pdf

[14] Opal-RT Technologies, "OP5600 off-the-shelf Hardware-in-the-Loop (HIL) simulator," [Online]. Available: http://http://www.opal-rt.com/ product/op5600-hil-hardware-in-the-loop-computer-and-IO-system

[15] R. S. Singh, H. Hooshyar and L. Vanfretti, "Assessment of Time Synchronization Requirements for Phasor Measurement Units", unpublished, submitted to IEEE PowerTech conference, 2015. 